\title{
The Effect of Diabetes on Mevalonate Metabolism in the Rat
}

\author{
M.H.Wiley, K. R. Feingold, M.M. Howton and M.D. Siperstein \\ Metabolism Section, Medical Service, Veterans Administration Medical Center, and Department of Medicine, University of California, \\ San Francisco, California, USA
}

\begin{abstract}
Summary. Previous studies have demonstrated a major difference in mevalonate metabolism by male and female rats by both the shunt and sterol pathways. This important sex difference was shown to be related to the presence of oestrogens and/or progesterone. In the present study we have investigated the effects of streptozotocin-induced diabetes on mevalonate metabolism. Firstly, insulin deficiency decreased the ability of the rats to oxidize mevalonate to carbon dioxide by the shunt pathway both in vivo and in vitro. This decrease was reversed by insulin treatment both in the intact animals as well as in tissue slices. Secondly, diabetes caused a marked increase in hepatic sterologenesis in the intact animal. This is the first demonstration that insulin plays a significant regulatory role in both the shunt and sterol pathways of mevalonate metabolism.
\end{abstract}

Key words: Diabetes, cholesterogenesis, mevalonate, insulin, mevalonate shunt, sterologenesis.

The synthesis of cholesterol de novo in man and other mammals is regulated primarily by the rate-limiting enzyme, 3-hydroxy-3-methylglutaryl coenzyme A (HMGCoA) reductase, the product of which is mevalonate. Mevalonate can be metabolized by two major mechanisms. Firstly, it can be converted by the sterol pathway to ubiquinone, dolichol and cholesterol; and secondly, mevalonate can be metabolized by a non-sterol (shunt) pathway which ultimately oxidizes it to carbon dioxide $[1,2]$. This alternative pathway, which diverts mevalonate from cholesterol production, can account for as much as $32 \%$ of the circulating mevalonate metabolized in vivo $[3,4]$.

In previous studies we have shown that physiological levels of circulating mevalonate are metabolized by both of these pathways primarily in the kidneys rather than in the liver. The kidney was found to me- tabolize circulating mevalonate in vivo by the sterol pathway at 20 times the rate of any other tissue [5]. We have also demonstrated that the kidney, both in vivo and in vitro, accounts for more than half of the total mevalonate oxidized to $\mathrm{CO}_{2}$ by the shunt pathway [3, $6,7]$. Similar results have been reported from other laboratories $[8,9]$.

Recent reports from this laboratory $[3,4,10]$ have demonstrated further that the female shunts mevalonate away from the sterol pathway at twice the rate of the male. This important sex difference was shown to result from the hormonal control of mevalonate metabolism by oestrogen and/or progesterone. The finding of sex hormonal regulation of mevalonate metabolism has stimulated studies to determine whether the mevalonate pathways are influenced by other hormonal factors. This study was designed to investigate the effect of diabetes on the metabolism of mevalonic acid to sterols and carbon dioxide in intact animals and in tissue slices.

\section{Materials and Methods}

\begin{abstract}
Materials
Materials were obtained as follows: $(R S)-\left[5-{ }^{14} \mathrm{C}\right] \mathrm{mevalonic}$ acid, sodium salt $(15 \mathrm{mCi} / \mathrm{mmol})$ from Research Products, Elkgrove, Illinois, USA; unlabelled $(R S)$-mevalonolactone from Sigma Chemicals, and streptozotocin from Sigma Chemicals, St. Louis, Missouri, USA and from Upjohn, Kalamazoo, Michigan, USA; U-I00 insulin from Eli Lilly, Indianapolis, Indiana, USA; thin layer polygram SilG plates from Brinkmann Instruments, Westbury, New York. Radio-autography was carried out on Kodak RP-14 X-ray film (Eastman Kodak, Rochester, New York). The liquid scintillation fluid contained scintillation-grade toluene obtained from Packard Corporation, Downers Grove, Illinois, USA; $1-\alpha, 2-\alpha$ tritiated cholesterol $(43 \mathrm{Ci} / \mathrm{mmol})$ and scintillation-grade PPO and POPOP were purchased from Amersham/Searle, Arlington Heights, Illinois, USA; and Bio-Solv III and Triton X-100 from Beckman Instruments, Palo Alto, California. The Alzet pumps were purchased from Alza, Palo Alto, California.
\end{abstract}


Table 1. Effect of diabetes and of insulin treatment on mevalonate oxidation in male rats

\begin{tabular}{lll}
\hline $\begin{array}{l}\text { Mevalonate oxidation to } \mathrm{CO}_{2}(\mathrm{nmol} / 6 \mathrm{~h}) \\
\text { Control } \\
(n=5)\end{array}$ & $\begin{array}{l}\text { Diabetic } \\
(n=6)\end{array}$ & $\begin{array}{l}\text { Insulin-treated } \\
(n=6)\end{array}$ \\
\hline $54.8 \pm 3.4$ & $28.8 \pm 2.1$ & $58.9 \pm 6.6$ \\
\hline
\end{tabular}

The values are expressed as mean \pm SEM. Numbers in parentheses refer to the number of animals tested

\section{Animals and Procedures}

Experimental methods used have been described previously. $[4,7]$ For studies in vivo, male Sprague-Dawley rats weighing between 200 and $400 \mathrm{~g}$ were maintained on a reverse $12-\mathrm{h}$ light cycle for one week before the study and were fed Berkeley rat and mouse diet and water ad libitum. The animals were fasted for $24 \mathrm{~h}$, after which they were injected IV with streptozotocin $(125 \mathrm{mg} / \mathrm{kg}$ rat weight $)$ dissolved in $0.1 \mathrm{~mol} / 1$ citrate buffer $(\mathrm{pH} 4.5)$ [11]. Only animals that developed $4+$ glycosuria were used in these studies; insulin-treated animals were given U-100 regular insulin (2-5U/day) by IP injection or by SC Alzet pump implants at the earliest evidence of glycosuria. All in vivo experiments were initiated 5-6 days after the streptozotocin injection. The animals were anaesthetized with diethyl ether, and $136 \mu \mathrm{g}$ of $\left[5-{ }^{14} \mathrm{C}\right]$ mevalonic acid in $0.4 \mathrm{ml}$ of $0.9 \% \mathrm{NaCl}$ was injected into the tail vein of each rat. For metabolic studies at approximately physiological levels, the concentration of $\left[5-{ }^{14} \mathrm{C}\right] \mathrm{mevalonic}$ acid was reduced to $8.5 \mu \mathrm{g}$. Following injection the animals were placed in 2-I widemouth Erlenmeyer flasks fitted with two-hole stoppers. Air was drawn through the flasks at the rate of $2 \mathrm{~V} / \mathrm{min}$, and expired ${ }^{14} \mathrm{CO}_{2}$ was collected in $1 \mathrm{~N} \mathrm{NaOH}$ solution $(2 \times 180 \mathrm{ml})$. At each hour, $0.2-\mathrm{ml}$ samples of the $\mathrm{NaOH}$ solution were added to $10 \mathrm{ml}$ of liquid scintillation fluid consisting of toluene $(1000 \mathrm{ml})$, Beckman Bio-Solv III $(300 \mathrm{ml})$, water $(100 \mathrm{ml})$, and PPO $(6 \mathrm{~g})$. The amount of ${ }^{14} \mathrm{CO}_{2}$ collected was determined on either a Beckman LS-330 or LS-230 liquid scintillation counter. After $6 \mathrm{~h}$ the rats were killed and the livers, kidneys and carcasses were saponified separately and analyzed for ${ }^{14} \mathrm{C}$-lipids as described below.

For the studies in vitro, female Sprague-Dawley rats weighing $200-400 \mathrm{~g}$ were maintained routinely on Berkeley rat and mouse chow and water ad libitum. Streptozotocin injection and insulin treatment were the same as described earlier. The animals were anaesthetized with diethyl ether and decapitated, after which the kidneys were rapidly removed and placed in Krebs-Ringer phosphate buffer. A whole kidney was sliced into ribbons on a McIlwain tissue slicer and a $200-\mathrm{mg}$ sample was placed in the outer well of a $25-\mathrm{ml}$ centre well flask containing $2 \mathrm{ml}$ of Krebs-Ringer phosphate buffer, $1 \mu \mathrm{Ci}$ of $\left[5{ }^{14} \mathrm{C}\right] \mathrm{mevalonate}$ and unlabelled mevalonate to yield a final concentration of $0.1 \mathrm{mmol} / 1$. The flasks were stoppered with serum caps and incubated at $37^{\circ} \mathrm{C}$ for $1 \mathrm{~h}$ in a Dubnoff metabolic shaker at $60 \mathrm{oscillations} / \mathrm{min}$. All experiments were carried out in duplicate.

At the end of the incubation period, $1 \mathrm{~N} \mathrm{NaOH}(0.9 \mathrm{ml})$ was injected through the serum caps into the centre wells, and $1 \mathrm{~N} \mathrm{H}_{2} \mathrm{SO}_{4}$ ( $\mathrm{l} \mathrm{ml}$ ) was injected into each outer well. ${ }^{14} \mathrm{CO}_{2}$ was collected in the centre wells by re-incubating the samples for $15 \mathrm{~min}$ in the Dubnoff shaker. Aliquots $(0.1 \mathrm{ml})$ of the centre well contents were added to $10 \mathrm{ml}$ of Beckman Bio-Solv III scintillation counting fluid previously described. All values were corrected for small amounts of volatile ${ }^{14} \mathrm{C}$ recovered from incubations with previously boiled tissues.

\section{Non-saponifiable Lipids}

After the addition of internal standards of tritiated cholesterol, the tissues to be studied were placed in Erlenmeyer flasks and refluxed overnight with $90 \% \mathrm{KOH}: 70 \%$ ethyl alcohol $(1: 5)$ as described previously $[4,7]$. The non-saponifiable material was extracted with petroleum ether $(50 \mathrm{ml})$. The petroleum ether was dried and dissolved in chloroform $(1 \mathrm{ml})$ and $100-\mu \mathrm{l}$ samples were applied to thin-layer chromatography plates. The plates were developed in ethyl acetate :benzene $(1: 5)$ for $50 \mathrm{~min}$, and radio-autographed on Kodak RP-14 X-ray film for 3-5 days. Areas of the plates corresponding to cholesterol, lanosterol and squalene markers were cut out and placed into vials containing $10 \mathrm{ml}$ counting solution consisting of toluene (1.331) Triton X-100 (0.661), PPO (9.33g) and POPOP $(0.133 \mathrm{~g})$. The gain and discriminator window settings of the scintillation counter were adjusted so that $<0.02 \%$ of the tritium counts were read in the ${ }^{14} \mathrm{C}$ window and approximately $15 \%$ of the ${ }^{14} \mathrm{C}$ counts were recorded in the tritium window. The amount of tritiated cholesterol added as internal standard was adjusted so that the tritium counts were approximately five times $>$ the ${ }^{14} \mathrm{C}$ counts. After correction for spillover of tritium and ${ }^{14} \mathrm{C}$ and for background radioactivity, counts were expressed as d.p.m. Although $(R S)-\left[5{ }^{14} \mathrm{C}\right] \mathrm{mevalonate}$ was used in this study, it is well established that only the $(R)$ isomer is metabolized [2]. For this reason all calculations assume that half of the administered mevalonate is inactive.

The histological sections, stained with haematoxylin and eosin, were prepared by standard techniques.

\section{Results}

\section{Effect of Diabetes and Insulin Therapy on the Mevalonate Shunt Pathway}

The initial experiments in vivo were concerned with determining the influence of diabetes and insulin therapy on the shunt pathway in the intact male animal. The metabolism of IV injected single doses of $\left[5-{ }^{14} \mathrm{C}\right]$ mevalonate $(136 \mu \mathrm{g})$ to ${ }^{14} \mathrm{CO}_{2}$ was monitored for 6-h periods in five control animals and in 12 male diabetic rats, one-half of whom were treated with insulin (Table 1). Diabetes suppressed the shunt activity in the animals by approximately $50 \%(p<0.001)$, but the activity was completely restored by insulin therapy (control versus treated diabetic rats $p>0.1$ ).

\section{Effects of Diabetes and Insulin Therapy on the Sterol Pathway}

These effects were studied in the kidneys, liver and carcass of the same rats used above. As shown in Figure $1 \mathrm{a}$, diabetes significantly influenced the accumulation of labelled sterols from ${ }^{14} \mathrm{C}$-mevalonate in the kidney. The average amount of mevalonate incorporated into sterols in the control male kidneys $(136 \mathrm{nmol} / 6 \mathrm{~h})$ was approximately twice that found in the kidneys of diabetic rats $(74 \mathrm{nmol} / 6 \mathrm{~h}, p<0.01)$. Insulin therapy did not significantly restore the synthesis of sterols in the kidney.

Diabetes caused an opposite change in hepatic sterols synthesized from circulating mevalonate (Figure $1 \mathrm{~b}$ ). The mean ${ }^{14} \mathrm{C}$-sterol synthesis in the diabetic male liver was twofold greater than that found in the control male liver ( 37 versus $18 \mathrm{nmol} / 6 \mathrm{~h}, p<0.01$ ).

Carcass sterols synthesized from circulating ${ }^{14} \mathrm{C}$ mevalonate increased in the diabetic rat $(50 \pm 4$ ver- 
(a) KIDNEY

(b) LIVER

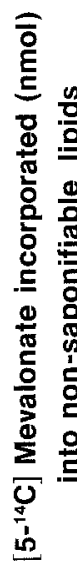

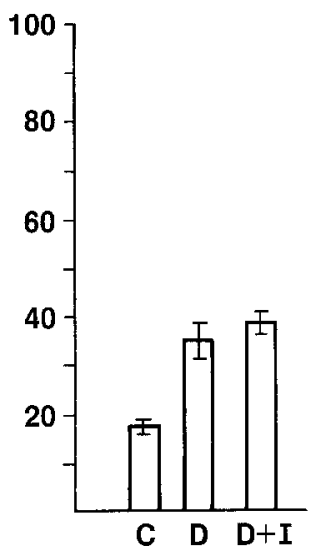

Fig. 1 a-b. Conversion of circulating $\left[5-{ }^{14} \mathrm{C}\right] \mathrm{mevalonate}$ in vivo to non-saponifiable lipids (squalene, lanosterol and cholesterol) in (a) the kidney and (b) the liver of male control $(C)$, diabetic $(D)$, and diabetic rats treated with insulin $(D+I)$. The rats were those used in the experiments shown in Table 1. Bars indicate mean values $\pm \mathrm{SEM}$

Table 2. Metabolism of $\left[5^{14} \mathrm{C}\right] \mathrm{mevalonate}$ in the liver of rats at physiological dose levels

\begin{tabular}{lll}
\hline Pathway & Control & Diabetic \\
\hline Shunt $\left(\right.$ nmol $\mathrm{CO}_{2}$ ) & 3.18 & 1.92 \\
Liver non-saponifiable lipids & 1.12 & 2.53 \\
(nmol) & & \\
\hline
\end{tabular}

Values represent mean values from duplicate animals

Table 3. $\left[5-{ }^{14} \mathrm{C}\right]$ mevalonate oxidation by rat kidney slices in vitro

\begin{tabular}{lll}
\hline \multicolumn{3}{l}{ Mevalonate oxidation to $\mathrm{CO}_{2}\left(\mathrm{nmol} \mathrm{g}^{-1} \mathrm{~h}^{-1}\right)$} \\
\hline Control & Diabetic & Insulin-treated \\
$24.1 \pm 1.6$ & $12.7 \pm 0.5$ & $28.9 \pm 1.8$
\end{tabular}

Each value represents the mean \pm SEM of duplicate determinations on six female rats

sus $84 \pm 9 \mathrm{nmol} / 6 \mathrm{~h} ; p<0.01)$. Insulin treatment did not significantly influence sterol synthesis in the carcass $(84 \pm 9$ versus $78 \pm 5 \mathrm{nmol} / 6 \mathrm{~h})$.

\section{The Effect of Substrate Concentration on Mevalonate} Metabolism in Vivo by the Shunt and Sterol Pathways

Studies were next carried out to determine whether a difference in mevalonate pool size between the diabetic and normal animals could account for the observed differences in mevalonate metabolism in the diabetic animal. The amount of mevalonate in the extracellular pool of a $200 \mathrm{~g}$ rat has previously been calculated by this laboratory to be about $2.4 \mu \mathrm{g}$ [5], a figure that is similar to that of Popják et al. [14]. We therefore studied the fate of mevalonate after injection of either $8.5 \mu \mathrm{g} /\left[5{ }^{-14} \mathrm{C}\right] \mathrm{m}$ evalonate, i.e. approximately a physiological dose, or $136 \mu \mathrm{g} /\left[5{ }^{14} \mathrm{C}\right] \mathrm{mevalo}-$ nate-approximately 15 times the physiological level. As shown in Table 2, using physiological doses, diabetes reduced the shunt activity and increased sterol production in the liver, thus confirming the observations using higher levels of injected mevalonate (Table 1 and Fig. 1).

\section{Metabolism of Mevalonate in Kidney Slices}

Since the kidney is the primary site of mevalonate metabolism by the shunt mechanism $[3,7,8]$, we evaluated the effect of diabetes on mevalonate metabolism by the kidney in vitro. As shown in Table 3, kidney slices from six normal animals metabolized [5$\left.{ }^{14} \mathrm{C}\right]$ mevalonate to ${ }^{14} \mathrm{CO}_{2}$ at approximately twice the rate of that found in kidney slices from diabetic rats $(p<0.001)$. Moreover, insulin treatment of the intact diabetic animals 2 days before study, completely restored the mevalonate shunt activity of the diabetic kidney.

Histological sections of the kidneys from the streptozotocin-treated rats showed minimal vacuolization of the tubular cells, with no evidence of interstitial changes. The glomeruli had no pathological changes. Insulin therapy appeared to decrease the tubular cell vacuolization slightly.

\section{Discussion}

The major finding of the present study is that insulin deficiency causes severe perturbations in both the sterol and shunt pathways of mevalonate metabolism. Streptozotocin diabetes markedly decreased the ability of the intact rat to divert mevalonate from sterol synthesis into the shunt pathway. The diabetic rat oxidized mevalonate to carbon dioxide by the shunt mechanism at only $50 \%$ of the normal rate.

Previous studies from this laboratory have demonstrated that the kidney is the primary site of mevalonate shunt activity in the rat [7]. When tissue slices were incubated with $\left[5-{ }^{14} \mathrm{C}\right]$ mevalonate, the insulindeficient kidney had approximately $50 \%$ of the control shunt activity. While quantitative extrapolations from results in vitro to in vivo must be made with caution, it seems reasonable that the influence of diabetes upon the mevalonate shunt pathway may be ascribed to a direct effect of insulinopenia on the renal metabolism of mevalonate. The possibility that the reduced shunt activity was due to nonspecific streptozotocin induced kidney damage was ruled out by two observations. Firstly, only minimal histological changes were detected in the kidneys and secondly, insulin 
treatment of the streptozotocin-diabetic rats caused a significant and rapid reversal of the depressed mevalonate shunt activity both in vivo and in vitro.

Shunt activity was consistently depressed in the diabetic rats, regardless of whether we administered approximately physiological or 15 times physiological levels of $\left[{ }^{14} \mathrm{C}\right]$ mevalonate. In addition, the effect of diabetes on mevalonate shunt metabolism was demonstrated by experiments in vitro, where activity in kidney slices of the insulin treated diabetic animals was restored to normal. These observations suggest that differences in mevalonate pool size as well as alterations in intermediate pools cannot be responsible for the decreased mevalonate shunt activity noted in the diabetic rat.

Concomitant with its effects on the shunt pathway, insulin deficiency had a pronounced influence on the sterol pathway of mevalonate metabolism in that diabetes increased its incorporation into sterols by twofold in the liver. This increase was not always reversed by insulin. However, it is well known that insulin has stimulatory effects on de novo cholesterol synthesis $[12,13]$. The mechanism by which diabetes consistently increased hepatic sterologenesis was not examined in the present study. However, we have demonstrated previously that removal of the kidneys, the major tissue site of shunt activity, causes a fivefold increase in the conversion of mevalonate to cholesterol in the liver. Thus, it is possible that when the renal shunt and sterol pathways are depressed by the diabetic state, mevalonate is channelled into the hepatic sterol pathway at an enhanced rate.

While the physiological significance of the shunt pathway is still unknown, we have demonstrated that mevalonate oxidation in normal human females is twice that of males [10]. Further, Fogelman et al. have shown that the shunt activity in a hypercholesterolaemic human male is approximately half that of a normal male [2]. Although the pathological implications of the increased hepatic conversion of mevalonate to sterols in diabetics have not yet been evaluated, this finding obviously correlates with the increased serum and liver cholesterol levels found in diabetic rats $[15,16]$ and man $[17,18]$. Studies are currently in progress to determine whether alterations in the pathways of mevalonate metabolism, comparable to those observed in the rat, are likewise present in human diabetes.

Acknowledgements. We appreciate the gift of streptozotocin from Dr. P. W.O'Connell, Upjohn Company; and we are grateful to Dr. C. G. Biava, Pathology Department, School of Medicine, University of California, San Francisco for the histological evaluation of the kidney sections. This work was supported by United States Public Health Service Program Project grant HL-06285 and grant CA15979 , by the Kroc Foundation, and by the Medical Research Service of the Veterans Administration. Dr. Feingold is the recipient of a Special Emphasis Research Career Award from the National In- stitutes of Health. A preliminary report of this study was presented to the American Society of Biological Chemists in Atlanta, Georgia on June 7, 1978 (Fed Proc $37: 1152$ ).

\section{References}

1. Edmond J, Popják G (1974) Transfer of carbon atoms from mevalonate to $n$ fatty acids. J Biol Chem 249:66-71

2. Fogelman A, Edmond J, Popják G (1975) Metabolism of mevalonate in rats and man not leading to sterols. J Biol Chem 250: 1771-1775

3. Wiley MH, Howton MM, Siperstein MD (1977) Renal metabolism of mevalonate: A sex difference. Fed Proc 36: 1817

4. Wiley MH, Howton MM, Siperstein MD (1978) Sex differences in the sterol and nonsterol metabolism of mevalonate. J Biol Chem 254: 837-842

5. Hellstrom KF, Siperstein MD, Bricker LA, Luby LJ (1973) Studies of the in vivo metabolism of mevalonic acid in the normal rat. J Clin Invest 52: 1303-1313

6. Righetti M, Wiley MH, Murrill PA, Siperstein MD (1976) The in vitro metabolism of mevalonate by sterol and nonsterol pathways. J Biol Chem 251:2716-2721

7. Wiley MH, Howton MM, Siperstein MD (1977) The quantitative role of the kidneys in the in vivo metabolism of mevalonate. J Biol Chem 252: 548-554

8. Edmond J, Fogelman AM, Popják G (1976) Mevalonate metabolism: role of the kidneys. Science 193: 154-156

9. Linder J, Beitz D (1978) Sterol synthesis and $\mathrm{CO}_{2}$ production from mevalonate in calves. J Lipid Res 19: 836-840

10. Feingold KR, Wiley MH, Searle G, Machida BK, Siperstein MD (1980) A sex difference in human mevalonate metabolism. J Clin Invest 66:361-366

11. Yum SI, Tillson SA, Theeuwes F (1976) Miniaturized osmotic pump technology for control of experimentally induced diabetes. In: James VNT (ed) Proceedings of the 5th International Congress of Endocrinology, Excerpta Medica, Amsterdam, p366

12. Bhathena S, Avigan J, Schreiner M (1974) Effect of insulin on sterol and fatty acid synthesis and hydroxy-methylglutaryl $\mathrm{CoA}$ reductase activity in mammalian cells grown in culture. Proc Natl Acad Sci USA 71:2174-2178

13. Frien BM, Saudek CD (1979) Cholesterol metabolism in diabetes: Effect of insulin on kinetics of plasma squalene. J Clin Endocrinol 49:824-829

14. Popják G, Boehm G, Parker TS, Edmond J, Edwards PA, Fogelman AM (1979) Determination of mevalonate in blood plasma in man and rat. Mevalonate tolerance tests in man. $\mathbf{J}$ Lipid Res 20:716-728

15. Nerui FL, Gonzalez A, Valdivieso V (1974) Studies on cholesterol metabolism in the diabetic rat. Metabolism 23:495-503

16. Bar-On H, Roheim P, Eder HA (1964) Hyperlipoproteinemia in streptozotocin-treated rats. Diabetes 25:509-515

17. Garcia MJ, McNamara PM, Gordon T, Kannel W (1974) Morbidity and mortality in diabetes in the Framingham population. Diabetes 23: 105-111

18. Chase HP, Glasgow AM (1976) Juvenile diabetes mellitus and serum lipids and lipoprotein levels. Am J Dis Child 130: $1113-1117$

Received: 12 January 1981

and in revised form: 29 September 1981

Dr. M.H.Wiley

Metabolism Section

VA Medical Center

4150 Clement Street (111F)

San Francisco, CA 94121, USA 